\title{
Short-Term Outcomes After Total Shoulder Arthroplasty in Octogenarians: A Matched Analysis
}

\author{
John Carney ${ }^{1}$, Erik Gerlach ${ }^{1}$, Mark A. Plantz ${ }^{1}$, Colin Cantrell ${ }^{1}$, Peter R. Swiatek ${ }^{2}$, Jeremy S. Marx ${ }^{1}$, \\ Guido Marra ${ }^{1}$ \\ 1. Department of Orthopaedic Surgery, Northwestern University Feinberg School of Medicine, Chicago, USA 2. \\ Department of Orthopaedic Surgery, Northwestern Memorial Hospital, Chicago, USA
}

Corresponding author: Erik Gerlach, erik.gerlach@northwestern.edu

\section{Abstract}

\section{Introduction}

Studies have shown that the use of total shoulder arthroplasty is increasing every year in the United Stated at a rate higher than that of total hip or total knee arthroplasty. As the population of the United States continues to age, it is becoming more important for surgeons to understand the true impact of age on outcomes and complications following procedures such a total shoulder arthroplasty. The purpose of this study was to determine if octogenarians have poorer outcomes after total shoulder arthroplasty compared to a younger, matched control group.

\section{Methods}

Data was obtained through the American College of Surgeons National Surgical Quality Improvement Program database (ACS NSQIP). Patients who had undergone total shoulder arthroplasty were identified by Current Procedural Terminology code (23472). Indication for arthroplasty was determined by ICD9/10 code (osteoarthritis, fracture, other). Each octogenarian was matched 1:1 to a non-octogenarian based on sex, BMI, ASA class, medical comorbidities, functional status, and surgical indication for arthroplasty by propensity scoring. A subgroup analysis was performed to compare outcomes between only those patients who underwent TSA for osteoarthritis. Outcomes of interest were assessed between the two groups for statistical significance using a chi-squared test or fisher exact test for expected values of less than 5 . Statistical significance was set at $\mathrm{p}<0.05$.

\section{Results}

After matching, octogenarians were found to be at higher risk of readmission ( $4.7 \%$ vs. $3.3 \%$, $p=0.046)$, nonhome discharge ( $27.1 \%$ vs. $9.4 \%, \mathrm{p}<0.001)$, and overall surgical $(4.4 \%$ vs. $2.5 \%, \mathrm{p}=0.006)$ and medical complications ( $3.7 \%$ vs. $2.4 \%, \mathrm{p}=0.039)$. In the setting of TSA for osteoarthritis only, however, octogenarians were only at higher risk for non-home discharge ( $22.4 \%$ vs. $7.5 \%$, $\mathrm{p}<0.001)$.

Review began 06/28/2021 Review ended 07/05/2021 Published 07/17/2021

\section{(c) Copyright 2021}

Carney et al. This is an open access article distributed under the terms of the Creative Commons Attribution License CC-BY 4.0., which permits unrestricted use, distribution, and reproduction in any medium, provided the original author and source are credited.

\section{Conclusions}

Octogenarians are at higher risk of some complications following total shoulder arthroplasty but fewer than has been previously reported, particularly in the setting of arthroplasty for the treatment of arthritis.

Categories: Orthopedics

Keywords: octogenarian, total shoulder arthroplasty, outcomes, risk factors, osteoarthritis, complications

\section{Introduction}

Total shoulder arthroplasty (TSA) has been well established as an efficacious and valid treatment modality for a myriad of glenohumeral joint pathologies [1-4]. The incidence of TSA has been growing annually in the United States and has currently outpaced that of total hip or total knee arthroplasty [5]. TSA is performed using one of two techniques. The first is the anatomic TSA technique which employs implants resembling native shoulder anatomy. The second being the reverse total shoulder arthroplasty (RTSA) technique that can function in the setting of rotator cuff insufficiency. This approach utilizes implants that places the ball component on the glenoid and the socket component to the proximal humerus [6]. A vast body of evidence shows that both TSA techniques have better radiographic and patient reported outcomes in comparison to other surgical treatment modalities for conditions such as proximal humerus fractures and glenohumeral arthritis $[2,3,7-9]$.

While TSA can provide excellent outcomes for patients with a variety of glenohumeral pathologies, the procedure is not risk free. Periprosthetic fracture, implant failure, peri-operative medical complications and 
infection are all serious complications of TSA that can lead to reoperation, diminished functional results, or even death if not managed in a timely and appropriate manner [10,11]. Hence, it is crucial that surgeons be meticulous in selecting appropriate patients for TSA and remain aware of risk factors for poor outcomes and complications.

Age has often been identified as a risk factor for complication throughout the orthopaedic literature [11-14]. However, this risk factor is non-modifiable, and with an aging population in the United States, there is a need for surgeons to better understand the impact of age on orthopaedic outcomes. This is particularly true in the setting of arthroplasty, which typically is performed in older populations. Octogenarians (i.e. individuals of age 80 or older) have been the subject of numerous outcome studies in arthroplasty which have shown this group to be at a higher risk of complication [11-13,15,16]. The purpose of this study was to determine if octogenarians have poorer outcomes after total shoulder arthroplasty compared to a younger, matched control group.

\section{Materials And Methods}

Data was obtained through the American College of Surgeons National Surgical Quality Improvement Program database (ACS NSQIP). This database is collected by a group of trained surgical clinical reviewers, who record perioperative data at over 700 hospitals across the United States, including International Classification of Disease 9th and/or 10th Revisions (ICD-9, ICD-10) codes, Current Procedural Terminology (CPT) codes, and data related to discharge disposition, reoperations, readmissions, and mortality through 30 days postoperatively [17]. This database is considered among the most precise and accurate database available for measuring patient outcomes after surgery given its high rate of complete data sets, operative data points, validation, and inter-rater reliability [18].

Patients who had undergone total shoulder arthroplasty were identified by CPT code (23472). Indication for arthroplasty was determined by ICD9/10 code (osteoarthritis, fracture, other). Patients were divided into two groups: octogenarians (80+ years old) and control (age 65-79). Both elective and non-elective procedures were included.

For the control group, each octogenarian was matched 1:1 to a non-octogenarian based on sex, BMI, ASA class, medical comorbidities, functional status, and surgical indication for arthroplasty. Matching was performed using propensity scoring. A subgroup analysis was performed to compare outcomes between only those patients who underwent TSA for a primary diagnosis of osteoarthritis.

Outcomes of interest included return to the operating room, readmission, reoperation, non-home discharge, mortality, surgical complications (superficial surgical site infection, deep surgical site infection, dehiscence, bleeding), medical complications (wound infection, pneumonia, reintubation, failure to wean intubation, pulmonary embolism, renal insufficiency, renal failure, urinary tract infection, cerebrovascular accident, cardiac arrest, myocardial infarction, deep venous thromboembolism, systemic sepsis, and septic shock). Differences in these outcomes were assessed between the two groups for statistical significance using a chisquared test or fisher exact test for expected values of less than 5 . Statistical significance was set at $\mathrm{p}<0.05$. All statistical calculations were performed using IBM SPSS Version 24 (Armonk, NY; IBM Corp).

\section{Results}

A total of 8,382 patients were eligible for inclusion in the study. Of these, 1,625 were octogenarians (mean age +/- SD) while the other 6,757 were aged 65-79 (mean age +/- SD). Prior to matching, the octogenarian group had lower rates of obesity (35.1\% vs. $51.2 \%$, p<0.001), diabetes $(15.9 \%$ vs. $20.1 \%, \mathrm{p}<0.001)$, and smoking $(2.8 \%$ vs. $7.2 \%, \mathrm{p}<0.001)$. The octogenarian group had higher rates of $\operatorname{COPD}(8.7 \%$ vs $6.9 \%, \mathrm{p}=0.012)$, CHF (1.2\% vs $0.6 \%$, p $=0.004$ ), hypertension ( $78.2 \%$ vs $71.1 \%$, $\mathrm{p}<0.001)$, bleeding disorders $(4.4 \%$ vs $2.4 \%$, $\mathrm{p}<0.001)$, and transfusions within 48 hours of surgery $(0.7 \%$ vs $0.2 \%, p=0.001)$. They were also less likely to be functionally independent $(94.6 \%$ vs $97.5 \%, \mathrm{p}<0.001$ ) and belong to an ASA class of 3 or greater $(64.6 \%$ vs $56.1 \%, \mathrm{p}<0.001)$. Lastly, octogenarians were more likely to undergo shoulder arthroplasty for fracture $(11.6 \%$ vs. $5.8 \%$, p <0.001) than osteoarthritis $(48.3 \%$ vs $55.0 \%$, $\mathrm{p}<0.001)$ compared to the control group $(\mathrm{p}<0.05)$. Findings are summarized in Table 1. 


\section{Cureus}

\begin{tabular}{|c|c|c|c|c|c|}
\hline & \multicolumn{2}{|c|}{$65-79$ years old } & \multicolumn{2}{|c|}{$80+$ years old } & \multirow{2}{*}{ p } \\
\hline & \multicolumn{2}{|c|}{$[n=6,757]$} & \multicolumn{2}{|c|}{$[n=1,625]$} & \\
\hline Male & 2,827 & $41.80 \%$ & 512 & $31.50 \%$ & $<0.001$ \\
\hline Female & 3,930 & $58.20 \%$ & 1,113 & $68.50 \%$ & \\
\hline \multicolumn{6}{|l|}{ BMI $\left(\mathrm{kg} / \mathrm{m}^{2}\right)$} \\
\hline Underweight & 82 & $1.20 \%$ & 29 & $1.80 \%$ & 0.071 \\
\hline Normal & 1,012 & $15.00 \%$ & 396 & $24.40 \%$ & $<0.001$ \\
\hline Overweight & 2,205 & $32.60 \%$ & 630 & $38.80 \%$ & $<0.001$ \\
\hline Obese Class I & 1,796 & $26.60 \%$ & 393 & $24.20 \%$ & 0.048 \\
\hline Obese Class II & 944 & $14.00 \%$ & 122 & $7.50 \%$ & $<0.001$ \\
\hline Obese Class III & 718 & $10.60 \%$ & 55 & $3.40 \%$ & $<0.001$ \\
\hline \multicolumn{6}{|l|}{ Comorbidities } \\
\hline Diabetes & 1,359 & $20.10 \%$ & 259 & $15.90 \%$ & $<0.001$ \\
\hline Smoking & 486 & $7.20 \%$ & 45 & $2.80 \%$ & $<0.001$ \\
\hline COPD & 468 & $6.90 \%$ & 142 & $8.70 \%$ & 0.012 \\
\hline Congestive Heart Failure & 38 & $0.60 \%$ & 20 & $1.20 \%$ & 0.004 \\
\hline Hypertension & 4,801 & $71.10 \%$ & 1,270 & $78.20 \%$ & $<0.001$ \\
\hline Renal Failure & 5 & $0.10 \%$ & 0 & $0.00 \%$ & 0.591 \\
\hline Chronic Steroid Use & 342 & $5.10 \%$ & 75 & $4.60 \%$ & 0.458 \\
\hline Bleeding Disorder & 162 & $2.40 \%$ & 72 & $4.40 \%$ & $<0.001$ \\
\hline Transfusion within 48 hours & 12 & $0.20 \%$ & 11 & $0.70 \%$ & 0.001 \\
\hline \multicolumn{6}{|l|}{ Functional Status } \\
\hline Independent & 6,591 & $97.50 \%$ & 1,538 & $94.60 \%$ & $<0.001$ \\
\hline Non-independent & 166 & $2.50 \%$ & 87 & $5.40 \%$ & \\
\hline \multicolumn{6}{|l|}{ Surgical Indication } \\
\hline Fracture & 389 & $5.80 \%$ & 189 & $11.60 \%$ & $<0.001$ \\
\hline Osteoarthritis & 3,717 & $55.00 \%$ & 785 & $48.30 \%$ & $<0.001$ \\
\hline Other & 2,651 & $39.20 \%$ & 651 & $40.10 \%$ & 0.54 \\
\hline \multicolumn{6}{|l|}{ ASA Class } \\
\hline Class 1 (No disturbance) & 71 & $1.10 \%$ & 3 & $0.20 \%$ & $<0.001$ \\
\hline Class 2 (Mild disturbance) & 2,704 & $40.00 \%$ & 499 & $30.70 \%$ & $<0.001$ \\
\hline Class 3 (Severe disturbance) & 3,788 & $56.10 \%$ & 1,050 & $64.60 \%$ & $<0.001$ \\
\hline Class 4+ (Life threatening) & 186 & $2.80 \%$ & 70 & $4.30 \%$ & 0.001 \\
\hline
\end{tabular}

TABLE 1: Demographics, patient variables, comorbidities, and surgical indication prior to matching

Prior to matching, the octogenarian group had higher rates of readmission ( $5.2 \%$ vs $2.8 \%, \mathrm{p}<0.001)$, nonhome discharge (30.0\% vs $9.9 \%, \mathrm{p}<0.001)$, mortality $(0.5 \%$ vs $0.1 \%, \mathrm{p}<0.001)$, surgical complications $(5.4 \%$ vs 
$2.4 \%, \mathrm{p}<0.001$ ), and medical complications (3.9\% vs $2.1 \%, \mathrm{p}<0.001)$. Regarding surgical complications specifically, the octogenarians had higher rates of bleeding (5.4\% vs. $2.0 \%$, p < 0.001$)$. Regarding medical complications, specifically, the octogenarians had higher rates of pneumonia ( $1.0 \%$ vs $0.5 \%, p=0.024)$, reintubation $(0.5 \%$ vs. $0.2 \%, \mathrm{p}=0.029)$, failure to wean intubation $(0.4 \%$ vs $0.1 \%, \mathrm{p}=0.007)$, pulmonary embolism ( $0.6 \%$ vs $0.3 \%$, p $=0.020)$, urinary tract infection (UTI) ( $1.5 \%$ vs $0.6 \%$, p $<0.001)$, cardiac arrest $(0.2 \%$ vs $0.0 \%, \mathrm{p}=0.015)$, and DVT $(0.6 \%$ vs $0.3 \%, \mathrm{p}=0.029)$. Findings are summarized in Table 2 .

\begin{tabular}{|c|c|c|c|c|c|}
\hline \multirow[b]{3}{*}{ Return to OR } & \multicolumn{2}{|c|}{$65-79$ years old } & \multicolumn{2}{|c|}{$80+$ years old } & \multirow{2}{*}{$\mathbf{p}$} \\
\hline & \multicolumn{2}{|c|}{$[n=6,757]$} & \multicolumn{2}{|c|}{$[n=1,625]$} & \\
\hline & 82 & $1.20 \%$ & 26 & $1.60 \%$ & 0.215 \\
\hline Readmission & 191 & $2.80 \%$ & 85 & $5.20 \%$ & $<0.001$ \\
\hline Reoperation & 82 & $1.20 \%$ & 26 & $1.60 \%$ & 0.215 \\
\hline Non-home discharge & 671 & $9.90 \%$ & 488 & $30.00 \%$ & $<0.001$ \\
\hline Mortality & 6 & $0.10 \%$ & 8 & $0.50 \%$ & $<0.001$ \\
\hline \multicolumn{6}{|l|}{ Surgical Complications } \\
\hline Overall & 161 & $2.40 \%$ & 88 & $5.40 \%$ & $<0.001$ \\
\hline Superficial surgical site infection & 10 & $0.10 \%$ & 1 & $0.10 \%$ & 0.703 \\
\hline Deep surgical site infection & 12 & $0.20 \%$ & 1 & $0.10 \%$ & 0.484 \\
\hline Dehiscence & 1 & $0.00 \%$ & 0 & $0.00 \%$ & $>0.999$ \\
\hline Bleeding & 138 & $2.00 \%$ & 87 & $5.40 \%$ & $<0.001$ \\
\hline \multicolumn{6}{|l|}{ Medical Complications } \\
\hline Overall & 144 & $2.10 \%$ & 64 & $3.90 \%$ & $<0.001$ \\
\hline Wound infection & 3 & $0.00 \%$ & 0 & $0.00 \%$ & $>0.999$ \\
\hline Pneumonia & 34 & $0.50 \%$ & 16 & $1.00 \%$ & 0.024 \\
\hline Reintubation & 13 & $0.20 \%$ & 8 & $0.50 \%$ & 0.029 \\
\hline Failure to wean intubation & 6 & $0.10 \%$ & 6 & $0.40 \%$ & 0.007 \\
\hline Pulmonary embolism & 17 & $0.30 \%$ & 10 & $0.60 \%$ & 0.02 \\
\hline Renal insufficiency & 5 & $0.10 \%$ & 2 & $0.10 \%$ & 0.627 \\
\hline Renal failure & 2 & $0.00 \%$ & 2 & $0.10 \%$ & 0.171 \\
\hline Urinary tract infection & 41 & $0.60 \%$ & 25 & $1.50 \%$ & $<0.001$ \\
\hline Cerebrovascular accident & 6 & $0.10 \%$ & 3 & $0.20 \%$ & 0.389 \\
\hline Cardiac arrest & 2 & $0.00 \%$ & 4 & $0.20 \%$ & 0.015 \\
\hline Myocardial infarction & 23 & $0.30 \%$ & 6 & $0.40 \%$ & 0.859 \\
\hline Deep venous thromboembolism & 18 & $0.30 \%$ & 10 & $0.60 \%$ & 0.029 \\
\hline Systemic sepsis & 10 & $0.10 \%$ & 5 & $0.30 \%$ & 0.171 \\
\hline Septic shock & 2 & $0.00 \%$ & 3 & $0.20 \%$ & 0.053 \\
\hline
\end{tabular}

TABLE 2: 30-day outcome measures and complication rates prior to matching

Propensity scoring resulted in the groups being well matched ( $p>0.99$ for all demographic and preoperative metrics) (Table 3). Compared to the matched control group, octogenarians continued to demonstrate significantly higher rates of readmission $(4.7 \%$ vs. $3.3 \%, \mathrm{p}=0.046)$ and non-home discharge $(27.1 \%$ vs $9.4 \%$, $\mathrm{p}<0.001)$. They also continued to demonstrate higher rates of overall surgical complications ( $4.4 \%$ vs $2.5 \%$, $\mathrm{p}=0.006)$ and bleeding ( $4.3 \%$ vs $2.2 \%, \mathrm{p}=0.001)$ as well as overall medical complications ( $3.7 \%$ vs $2.4 \%$, 


\section{Cureus}

$\mathrm{p}=0.039)$. However, they no longer demonstrated statistically different rates of mortality or any specific medical complication (Table 4). Subgroup analysis of octogenarians undergoing shoulder arthroplasty for a diagnosis of osteoarthritis only demonstrated a significantly higher rate of non-home discharge $(22.4 \%$ vs $7.5 \%, \mathrm{p}<0.001$ ) (Table 5). 


\section{Cureus}

\begin{tabular}{|c|c|c|c|c|c|}
\hline & \multicolumn{2}{|c|}{$65-79$ years old } & \multicolumn{2}{|c|}{$80+$ years old } & \multirow{2}{*}{ p } \\
\hline & \multicolumn{2}{|c|}{$[n=1,434]$} & \multicolumn{2}{|c|}{$[n=1,434]$} & \\
\hline Male & 454 & $31.70 \%$ & 454 & $31.70 \%$ & $>0.999$ \\
\hline Female & 980 & $68.30 \%$ & 980 & $68.30 \%$ & \\
\hline \multicolumn{6}{|l|}{ BMI $\left(\mathrm{kg} / \mathrm{m}^{2}\right)$} \\
\hline Underweight & 16 & $1.10 \%$ & 16 & $1.10 \%$ & $>0.999$ \\
\hline Normal & 328 & $22.90 \%$ & 328 & $22.90 \%$ & $>0.999$ \\
\hline Overweight & 575 & $40.10 \%$ & 575 & $40.10 \%$ & $>0.999$ \\
\hline Obese Class I & 355 & $24.80 \%$ & 355 & $24.80 \%$ & $>0.999$ \\
\hline Obese Class II & 112 & $7.80 \%$ & 112 & $7.80 \%$ & $>0.999$ \\
\hline Obese Class III & 48 & $3.30 \%$ & 48 & $3.30 \%$ & $>0.999$ \\
\hline \multicolumn{6}{|l|}{ Comorbidities } \\
\hline Diabetes & 222 & $15.50 \%$ & 222 & $15.50 \%$ & $>0.999$ \\
\hline Smoker & 33 & $2.30 \%$ & 33 & $2.30 \%$ & $>0.999$ \\
\hline COPD & 91 & $6.30 \%$ & 91 & $6.30 \%$ & $>0.999$ \\
\hline Congestive Heart Failure & 1 & $0.10 \%$ & 1 & $0.10 \%$ & $>0.999$ \\
\hline Hypertension & 1,127 & $78.60 \%$ & 1,127 & $78.60 \%$ & $>0.999$ \\
\hline Renal Failure & 0 & $0.00 \%$ & 0 & $0.00 \%$ & $>0.999$ \\
\hline Chronic Steroid Use & 47 & $3.30 \%$ & 47 & $3.30 \%$ & $>0.999$ \\
\hline Bleeding Disorder & 35 & $2.40 \%$ & 35 & $2.40 \%$ & $>0.999$ \\
\hline Transfusion within 48 hours & 1 & $0.10 \%$ & 1 & $0.10 \%$ & $>0.999$ \\
\hline \multicolumn{6}{|l|}{ Functional status } \\
\hline Independent & 1,404 & $97.90 \%$ & 1,404 & $97.90 \%$ & $>0.999$ \\
\hline Non-independent & 30 & $2.10 \%$ & 30 & $2.10 \%$ & $>0.999$ \\
\hline \multicolumn{6}{|l|}{ Surgical Indication } \\
\hline Fracture & 119 & $8.30 \%$ & 119 & $8.30 \%$ & $>0.999$ \\
\hline Osteoarthritis & 731 & $51.00 \%$ & 731 & $51.00 \%$ & $>0.999$ \\
\hline Other & 584 & $40.70 \%$ & 584 & $40.70 \%$ & $>0.999$ \\
\hline \multicolumn{6}{|l|}{ ASA Class } \\
\hline Class 1 (No disturbance) & 3 & $0.20 \%$ & 3 & $0.20 \%$ & $>0.999$ \\
\hline Class 2 (Mild disturbance) & 471 & $32.80 \%$ & 471 & $32.80 \%$ & $>0.999$ \\
\hline Class 3 (Severe disturbance) & 937 & $65.30 \%$ & 937 & $65.30 \%$ & $>0.999$ \\
\hline Class 4+ (Life threatening) & 23 & $1.60 \%$ & 23 & $1.60 \%$ & $>0.999$ \\
\hline
\end{tabular}

TABLE 3: Demographics, patient variables, comorbidities, and surgical indication after matching 


\section{Cureus}

\begin{tabular}{|c|c|c|c|c|c|}
\hline \multirow[b]{3}{*}{ Return to OR } & \multicolumn{2}{|c|}{$65-79$ years old } & \multicolumn{2}{|c|}{$80+$ years old } & \multirow{2}{*}{$\mathbf{p}$} \\
\hline & \multicolumn{2}{|c|}{$[n=1,434]$} & \multicolumn{2}{|c|}{$[n=1,434]$} & \\
\hline & 19 & $1.30 \%$ & 22 & $1.50 \%$ & 0.637 \\
\hline Readmission & 47 & $3.30 \%$ & 68 & $4.70 \%$ & 0.046 \\
\hline Reoperation & 19 & $1.30 \%$ & 22 & $1.50 \%$ & 0.637 \\
\hline Non-home discharge & 135 & $9.40 \%$ & 389 & $27.10 \%$ & $<0.001$ \\
\hline Mortality & 1 & $0.10 \%$ & 6 & $0.40 \%$ & 0.125 \\
\hline \multicolumn{6}{|l|}{ Surgical Complications } \\
\hline Overall & 36 & $2.50 \%$ & 63 & $4.40 \%$ & 0.006 \\
\hline Superficial surgical site infection & 2 & $0.10 \%$ & 1 & $0.10 \%$ & $>0.999$ \\
\hline Deep surgical site infection & 3 & $0.20 \%$ & 1 & $0.10 \%$ & 0.625 \\
\hline Dehiscence & 0 & $0.00 \%$ & 0 & $0.00 \%$ & - \\
\hline Bleeding & 31 & $2.20 \%$ & 62 & $4.30 \%$ & 0.001 \\
\hline \multicolumn{6}{|l|}{ Medical Complications } \\
\hline Overall & 34 & $2.40 \%$ & 53 & $3.70 \%$ & 0.039 \\
\hline Wound infection & 0 & $0.00 \%$ & 0 & $0.00 \%$ & - \\
\hline Pneumonia & 8 & $0.60 \%$ & 13 & $0.90 \%$ & 0.273 \\
\hline Reintubation & 4 & $0.30 \%$ & 7 & $0.50 \%$ & 0.548 \\
\hline Failure to wean intubation & 2 & $0.10 \%$ & 6 & $0.40 \%$ & 0.288 \\
\hline Pulmonary embolism & 3 & $0.20 \%$ & 9 & $0.60 \%$ & 0.145 \\
\hline Renal insufficiency & 1 & $0.10 \%$ & 2 & $0.10 \%$ & $>0.999$ \\
\hline Renal fallure & 0 & $0.00 \%$ & 2 & $0.10 \%$ & 0.5 \\
\hline Urinary tract infection & 10 & $0.70 \%$ & 20 & $1.40 \%$ & 0.066 \\
\hline Cerebrovascular accident & 1 & $0.10 \%$ & 3 & $0.20 \%$ & 0.625 \\
\hline Cardiac arrest & 0 & $0.00 \%$ & 3 & $0.20 \%$ & 0.25 \\
\hline Myocardial infarction & 6 & $0.40 \%$ & 5 & $0.30 \%$ & 0.763 \\
\hline Deep venous thromboembolism & 2 & $0.10 \%$ & 8 & $0.60 \%$ & 0.109 \\
\hline Systemic sepsis & 4 & $0.30 \%$ & 4 & $0.30 \%$ & $>0.999$ \\
\hline Septic shock & 1 & $0.10 \%$ & 3 & $0.20 \%$ & 0.625 \\
\hline
\end{tabular}

TABLE 4: 30-day outcome measures and complication rates after matching 


\section{Cureus}

\begin{tabular}{|c|c|c|c|c|c|}
\hline \multirow[b]{3}{*}{ Return to OR } & \multirow{2}{*}{\multicolumn{2}{|c|}{$\begin{array}{l}\text { 65-79 years old } \\
{[n=731]}\end{array}$}} & \multirow{2}{*}{\multicolumn{2}{|c|}{$\begin{array}{l}\text { 80+ years old } \\
{[n=731]}\end{array}$}} & \multirow{3}{*}{$\begin{array}{l}\mathbf{p} \\
0.807\end{array}$} \\
\hline & & & & & \\
\hline & 8 & $1.10 \%$ & 9 & $1.20 \%$ & \\
\hline Readmission & 17 & $2.30 \%$ & 22 & $3.00 \%$ & 0.417 \\
\hline Reoperation & 8 & $1.10 \%$ & 9 & $1.20 \%$ & 0.807 \\
\hline Non-home discharge & 55 & $7.50 \%$ & 164 & $22.40 \%$ & $<0.001$ \\
\hline Mortality & 0 & $0.00 \%$ & 4 & $0.50 \%$ & 0.124 \\
\hline \multicolumn{6}{|l|}{ Surgical Complications } \\
\hline Overall & 11 & $1.50 \%$ & 15 & $2.10 \%$ & 0.429 \\
\hline Superficial surgical site infection & 1 & $0.10 \%$ & 0 & $0.00 \%$ & $>0.999$ \\
\hline Deep surgical site infection & 1 & $0.10 \%$ & 0 & $0.00 \%$ & $>0.999$ \\
\hline Dehiscence & 0 & $0.00 \%$ & 0 & $0.00 \%$ & - \\
\hline Bleeding & 9 & $1.20 \%$ & 15 & $2.10 \%$ & 0.217 \\
\hline \multicolumn{6}{|l|}{ Medical Complications } \\
\hline Overall & 15 & $2.10 \%$ & 21 & $2.90 \%$ & 0.311 \\
\hline Wound infection & 0 & $0.00 \%$ & 0 & $0.00 \%$ & - \\
\hline Pneumonia & 4 & $0.50 \%$ & 4 & $0.50 \%$ & $>0.999$ \\
\hline Reintubation & 1 & $0.10 \%$ & 2 & $0.30 \%$ & $>0.999$ \\
\hline Failure to wean intubation & 1 & $0.10 \%$ & 3 & $0.40 \%$ & 0.624 \\
\hline Pulmonary embolism & 1 & $0.10 \%$ & 5 & $0.70 \%$ & 0.218 \\
\hline Renal insufficiency & 1 & $0.10 \%$ & 2 & $0.30 \%$ & $>0.999$ \\
\hline Renal failure & 0 & $0.00 \%$ & 0 & $0.00 \%$ & - \\
\hline Urinary tract infection & 5 & $0.70 \%$ & 6 & $0.80 \%$ & 0.762 \\
\hline Cerebrovascular accident & 1 & $0.10 \%$ & 1 & $0.10 \%$ & $>0.999$ \\
\hline Cardiac arrest & 0 & $0.00 \%$ & 1 & $0.10 \%$ & $>0.999$ \\
\hline Myocardial infarction & 2 & $0.30 \%$ & 2 & $0.30 \%$ & $>0.999$ \\
\hline Deep venous thromboembolism & 0 & $0.00 \%$ & 2 & $0.30 \%$ & 0.5 \\
\hline Systemic sepsis & 3 & $0.40 \%$ & 0 & $0.00 \%$ & 0.249 \\
\hline Septic shock & 1 & $0.10 \%$ & 1 & $0.10 \%$ & $>0.999$ \\
\hline
\end{tabular}

TABLE 5: 30-day outcome measures and complication rates after elective rTSA for osteoarthritis: matched comparison

\section{Discussion}

The purpose of this study was to determine if octogenarians have inferior short-term outcomes after total shoulder arthroplasty compared to a younger, matched control group. After matching, our data shows that octogenarians are at higher risk of readmission, non-home discharge, and surgical and medical complication. In the setting of TSA for osteoarthritis, however, octogenarians do not have a higher rate of readmission or complication but continue to have higher rates of non-home discharge.

To our knowledge there are two other studies that has investigated TSA in octogenarians. One study similarly found a higher overall complication rate in octogenarians. However, this group also found that octogenarians were at a higher risk of several, specific major and minor complications within 30 days 
including pulmonary embolism, stroke, myocardial infarction, reintubation and septic shock [11]. The differences between our results and the aforementioned study are likely due to the fact that the aforementioned study did not create two equal sized groups, but rather compared all TSA patients in the database for a set time period. Like in our sample, the octogenarians and non-octogenarians in this study had several significant differences in demographics and comorbidities. The aforementioned study accounted for these differences by utilizing a logistic regression model whereas our study created a 1:1 matched control group via propensity scoring, which may better account for differences in covariates among two groups and explain why we did not find octogenarians to be at a higher risk for these major complications. The other study also utilized the same database as ours and found that octogenarians were at a higher risk for readmission, pneumonia, and UTI compared to individuals younger than 70 years old. They were not, however, at a higher risk for any complications than individuals aged 70-80 [19].

Several studies have been conducted on octogenarians in the hip, knee, and ankle arthroplasty fields that are in agreement with our findings. A recent database study of octogenarians undergoing ankle arthroplasty found the group to be at a higher risk for non-home discharge and longer hospital length of stay but not at an increased risk for higher complication [12]. A 2016 review article that compiled morbidity and mortality data from 11 different studies on hip and knee arthroplasty in octogenarians and nonagenarians concluded that although advanced age patients have more medical comorbidities, these comorbidities do not translate to a higher rate of surgical complication, though some studies did note higher rates of medical complications [20-26]. Although our study did find a higher rate of complications in octogenarians, this finding became statistically insignificant when TSA patients without a primary diagnosis of osteoarthritis were removed from the dataset, which is likely a more similar patient population to the hip and knee arthroplasty populations. Some studies have found longer lengths of stay in this population following arthroplasty. More recent literature in advanced age patients, however, has not found this to be the case, which can likely be attributed to improved protocols involving co-management of older patients with geriatricians $[20,24]$.

Similar findings have also been found when assessing the revision arthroplasty in octogenarians. A 2007 study investigating outcomes in revision hip arthroplasty patients over the age of 80 compared to a control group found equivalent patient reported outcome scores and no difference in complications other than dislocation, which was higher in the younger control group [27]. A 2018 study investigating total knee arthroplasty in octogenarians compared to a propensity score matched control group found higher rate of blood transfusion and length of stay, but no increases in any other medical or surgical complication nor mortality or readmission [13].

This study has several weaknesses which should be considered when interpreting our findings. First, this study utilizes a database which can be subject to input errors and can only provide a limited number of metrics regarding a patient's medical care. The database does not provide specific information regarding severity of comorbidities or specific outcomes related to a given procedure. Access to the database is limited to contributing institutions, and the high cost of participation has led to a disproportionate contribution to the NSQIP from large teaching hospitals [18,28]. Second, the database identifies all-cause readmission, not just orthopedic-related readmissions. Third, there are no separate CPT codes for anatomic total shoulder arthroplasty or reverse total shoulder arthroplasty, and thus we are not able to identify whether the groups were equally matched regarding the type of arthroplasty that was performed, or if one form of arthroplasty demonstrated lower complication rates.

This study contributes to the current body of literature as it presents valuable information to surgeons on the increased risk that age alone presents when performing total shoulder arthroplasty, and can help surgeons make appropriate decisions regarding how to care for patients of this age group. Further, a prospective study is warranted to better quantify what factors are predictive of better or poorer outcomes in this population.

\section{Conclusions}

Octogenarians are at higher risk of readmission, non-home discharge, and surgical and medical complications following total shoulder arthroplasty. However, this age group is only at risk of non-home discharge when TSA is being performed for the diagnosis of arthritis. This information will assist surgeons with appropriately selecting surgical candidates in their practice and anticipating potential challenges and complications in the early post-operative period.

\section{Additional Information}

\section{Disclosures}

Human subjects: All authors have confirmed that this study did not involve human participants or tissue. Animal subjects: All authors have confirmed that this study did not involve animal subjects or tissue. Conflicts of interest: In compliance with the ICMJE uniform disclosure form, all authors declare the following: Payment/services info: All authors have declared that no financial support was received from any organization for the submitted work. Financial relationships: All authors have declared that they have 
no financial relationships at present or within the previous three years with any organizations that might have an interest in the submitted work. Other relationships: All authors have declared that there are no other relationships or activities that could appear to have influenced the submitted work.

\section{References}

1. Roberson TA, Bentley JC, Griscom JT, Kissenberth MJ, Tolan SJ, Hawkins RJ, Tokish JM: Outcomes of total shoulder arthroplasty in patients younger than 65 years: a systematic review. J Shoulder Elbow Surg. 2017, 26:1298-306. 10.1016/j.jse.2016.12.069

2. Cuff DJ, Pupello DR: Comparison of hemiarthroplasty and reverse shoulder arthroplasty for the treatment of proximal humeral fractures in elderly patients. J Bone Joint Surg Am. 2013, 95:2050-5. 10.2106/JBJS.L.01637

3. Craig RS, Goodier H, Singh JA, Hopewell S, Rees JL: Shoulder replacement surgery for osteoarthritis and rotator cuff tear arthropathy. Cochrane Database Syst Rev. 2020, 4:CD012879.

10.1002/14651858.CD012879.pub2

4. Hyun YS, Huri G, Garbis NG, McFarland EG: Uncommon indications for reverse total shoulder arthroplasty . Clin Orthop Surg. 2013, 5:243-55. 10.4055/cios.2013.5.4.243

5. Wagner ER, Farley KX, Higgins I, Wilson JM, Daly CA, Gottschalk MB: The incidence of shoulder arthroplasty: rise and future projections compared with hip and knee arthroplasty. J Shoulder Elbow Surg. 2020, 29:2601-9. 10.1016/j.jse.2020.03.049

6. Berliner JL, Regalado-Magdos A, Ma CB, Feeley BT: Biomechanics of reverse total shoulder arthroplasty. J Shoulder Elbow Surg. 2015, 24:150-60. 10.1016/j.jse.2014.08.003

7. Austin DC, Torchia MT, Cozzolino NH, Jacobowitz LE, Bell JE: Decreased reoperations and improved outcomes with reverse total shoulder arthroplasty in comparison to hemiarthroplasty for geriatric proximal humerus fractures: a systematic review and meta-analysis. J Orthop Trauma. 2019, 33:49-57. 10.1097/BOT.0000000000001321

8. Osterhoff G, O'Hara NN, D'Cruz J, Sprague SA, Bansback N, Evaniew N, Slobogean GP: A cost-effectiveness analysis of reverse total shoulder arthroplasty versus hemiarthroplasty for the management of complex proximal humeral fractures in the elderly. Value Health. 2017, 20:404-11. 10.1016/j.jval.2016.10.017

9. Stanbury SJ, Voloshin I: Reverse shoulder arthroplasty for acute proximal humeral fractures in the geriatric patient: a review of the literature. Geriatr Orthop Surg Rehabil. 2011, 2:181-6. 10.1177/2151458511420140

10. Somerson JS, Hsu JE, Neradilek MB, Matsen FA 3rd: Analysis of 4063 complications of shoulder arthroplasty reported to the US Food and Drug Administration from 2012 to 2016. J Shoulder Elbow Surg. 2018, 27:197886. 10.1016/j.jse.2018.03.025

11. Newman JM, Stroud SG, Yang A, et al.: Total shoulder arthroplasty in octogenarians: Is there a higher risk of adverse outcomes?. J Orthop. 2018, 15:671-5. 10.1016/j.jor.2018.05.035

12. Partan M, Frane N, Iturriaga C, Matai P, Bitterman A: Short-term outcomes of primary total ankle arthroplasty in octogenarians: a national database analysis. Foot Ankle Spec. 2020, 1938640020960546. $10.1177 / 1938640020960546$

13. Bovonratwet P, Tyagi V, Ottesen TD, Ondeck NT, Rubin LE, Grauer JN: Revision total knee arthroplasty in octogenarians: an analysis of 957 cases. J Arthroplasty. 2018, 33:178-84. 10.1016/j.arth.2017.07.032

14. Elsiwy Y, Jovanovic I, Doma K, Hazratwala K, Letson H: Risk factors associated with cardiac complication after total joint arthroplasty of the hip and knee: a systematic review. J Orthop Surg Res. 2019, 14:15. 10.1186/s13018-018-1058-9

15. Mednick RE, Alvi HM, Krishnan V, Lovecchio F, Manning DW: Factors affecting readmission rates following primary total hip arthroplasty. J Bone Joint Surg Am. 2014, 96:1201-9. 10.2106/JBJS.M.00556

16. Joshi AB, Markovic L, Gill G: Knee arthroplasty in octogenarians: results at 10 years . J Arthroplasty. 2003, 18:295-8. 10.1054/arth.2003.50063

17. ACS National Surgical Quality Improvement Program. (2020). Accessed: November 25, 2020: https://www.facs.org/Quality-Programs/ACS-NSQIP..

18. Alluri RK, Leland H, Heckmann N: Surgical research using national databases. Ann Transl Med. 2016, 4:393. 10.21037/atm.2016.10.49

19. Bovonratwet P, Malpani R, Ondeck NT, Tyagi V, Grauer JN: Elective total shoulder arthroplasty in octogenarians: a safe procedure. J Am Acad Orthop Surg. 2019, 27:145-54. 10.5435/JAAOS-D-17-00364

20. Rubin LE, Blood TD, Defillo-Draiby JC: Total hip and knee arthroplasty in patients older than age 80 years . J Am Acad Orthop Surg. 2016, 24:683-90. 10.5435/JAAOS-D-15-00278

21. Alfonso DT, Howell RD, Strauss EJ, Di Cesare PE: Total hip and knee arthroplasty in nonagenarians. J Arthroplasty. 2007, 22:807-11. 10.1016/j.arth.2006.10.016

22. Krishnan E, Fries JF, Kwoh CK: Primary knee and hip arthroplasty among nonagenarians and centenarians in the United States. Arthritis Rheum. 2007, 57:1038-42. 10.1002/art.22888

23. de Thomasson E, Caux I, Guingand O, Terracher R, Mazel C: Total hip arthroplasty for osteoarthritis in patients aged 80 years or older: influence of co-morbidities on final outcome. Orthop Traumatol Surg Res. 2009, 95:249-53. 10.1016/j.otsr.2009.03.011

24. Miric A, Inacio MC, Kelly MP, Namba RS: Are nonagenarians too old for total hip arthroplasty? an evaluation of morbidity and mortality within a total joint replacement registry. J Arthroplasty. 2015, 30:1324-7. 10.1016/j.arth.2015.03.008

25. D'Apuzzo MR, Pao AW, Novicoff WM, Browne JA: Age as an independent risk factor for postoperative morbidity and mortality after total joint arthroplasty in patients 90 years of age or older. J Arthroplasty. 2014, 29:477-80. 10.1016/j.arth.2013.07.045

26. Petruccelli D, Rahman WA, de Beer J, Winemaker M: Clinical outcomes of primary total joint arthroplasty among nonagenarian patients. J Arthroplasty. 2012, 27:1599-603. 10.1016/i.arth.2012.03.007

27. Parvizi J, Pour AE, Keshavarzi NR, D'Apuzzo M, Sharkey PF, Hozack WJ: Revision total hip arthroplasty in octogenarians. A case-control study. J Bone Joint Surg Am. 2007, 89:2612-8. 10.2106/JBJS.F.00881

28. Sheils CR, Dahlke AR, Kreutzer L, Bilimoria KY, Yang AD: Evaluation of hospitals participating in the American College of Surgeons National Surgical Quality Improvement Program. Surgery. 2016, 160:1182-8. 


\section{Cureus}

10.1016/j.surg.2016.04.034 